RESENDE, G.M.; COSTA, N.D. Produtividade e armazenamento de cebola, cv. Alfa Tropical, cultivada em diferentes espaçamentos. Horticultura Brasileira, Brasília, v.23, n.4, p.1010-1014, out-dez 2005.

\title{
Produtividade e armazenamento de cebola cv. Alfa Tropical cultivada em diferentes espaçamentos
}

\author{
Geraldo M. de Resende; Nivaldo Duarte Costa \\ Embrapa Semi-Árido, C. Postal 23, 56300-970 Petrolina-PE; E-mail: gmilanez@cpatsa.embrapa.br
}

\begin{abstract}
RESUMO
Com o objetivo de avaliar o efeito de diferentes espaçamentos entrelinhas e entre plantas sobre as características produtivas e o armazenamento de bulbos de cebola, conduziu-se um experimento de setembro de 1999 a março de 2000, em Petrolina. O delineamento experimental utilizado foi de blocos ao acaso, no esquema fatorial $2 \times 3$, compreendendo dois espaçamentos entrelinhas $(0,10$ e $0,15 \mathrm{~m})$ e três espaçamentos entre plantas $(0,10,0,20$ e $0,30 \mathrm{~m})$, sendo utilizada a cultivar Alfa Tropical com quatro repetições. Foram encontradas reduções lineares na produtividade comercial com o aumento do espaçamento entre plantas, tendo o espaçamento com $0,10 \mathrm{~m}$ propiciado os maiores rendimentos. Uma redução gradativa na produção de bulbos não comerciais foi verificada à medida que se aumentou o espaçamento entrelinhas e plantas. A massa fresca dos bulbos aumentou linearmente à medida que se aumentaram os espaçamentos entrelinhas e entre plantas. Foi verificada maior porcentagem de bulbos pequenos e médios nos menores espaçamentos. À medida que se incrementou o espaçamento entre plantas ocorreu maior perda de massa fresca dos bulbos de cebola até 60 dias de armazenamento. Pelos resultados obtidos em função das diferentes características avaliadas, recomenda-se os espaçamentos de $0,10 \mathrm{ou}$ $0,15 \mathrm{~m}$ entrelinhas e $0,10 \mathrm{~m}$ entre plantas como os mais adequados para o cultivo da cebola cultivar Alfa Tropical, nas condições do Vale do São Francisco, para plantio no segundo semestre do ano.
\end{abstract}

Palavras-chave: Allium cepa, rendimento, densidade de plantio, perda de massa fresca de bulbos.

\begin{abstract}
Yield and storage of onion, cv. Alfa Tropical, under different planting spacings

The effect of different plant spacing was evaluated on yield and storage characteristics of onion bulbs. The experiment was carried out from September 1999 to March 2000, in Pernambuco State, Brazil, in a randomized complete block design, in a 2 × 3 factorial scheme, with four replications. The cultivar Alfa Tropical was planted at 0.10 and $0.15 \mathrm{~m}$ row spacing and at $0.10 ; 0.15$ and $0.30 \mathrm{~m}$ plant spacing. A linear reduction was found for commercial yield when plant spacing increased, the highest yield being obtained with 0.10 $\mathrm{m}$ spacing. A linear reduction in noncommercial bulbs was found as the spacing between rows and between plants was increased. Bulbs fresh weight increased linearly as the spacing between rows and between plants increased. Larger percentage of small and medium bulbs was obtained in the narrowest spacing. As spacing between plants increased, a larger lose of onion bulb weight was found up to 60 days of storage. According to the results, the spacing $0.10 \times 0.10$ $\mathrm{m}$ or $0,15 \times 0.10 \mathrm{~m}$ are recommended as the more adapted for the cultivation of onion cultivar Alfa Tropical, in the São Francisco Valley conditions, for the summer season planting.
\end{abstract}

Keywords: Allium cepa, yield, fresh mass of bulb, planting density, bulb mass fresh loss.

\section{(Recebido para publicação em 11 de março de 2005 e aceito em 3 de agosto de 2005)}

A produção mundial de cebola (Allium cepa L.), em 2004, foi de 53,59 milhões de $t$, cultivadas em uma área 3,07 milhões de ha, o que proporcionou uma produtividade média de 17,46 t ha ${ }^{-1}$ (FAO, 2005). No Brasil, a cebola ocupa o terceiro lugar em importância econômica entre as hortaliças (SOUZA; RESENDE, 2002). A produtividade média foi de $17,88 \mathrm{t} \mathrm{ha}^{-1} \mathrm{em}$ 2004, sendo que nos estados de PE e BA, maiores produtores do Nordeste, a produtividade média foi de 21,16 e 24,25 t ha $^{-1}$, respectivamente (IBGE, 2005).

A cebola é planta de dias longos quanto à formação de bulbos, e as cultivares designadas de dias curtos não são, particularmente, plantas de dias curtos; simplesmente exigem menos horas de luz para bulbificarem (MELO; RIBEI-
RO, 1990). A formação de bulbos está relacionada com a interação entre a temperatura e o fotoperíodo. Nesta interação o fator mais importante é o fotoperíodo e o mesmo determina os limites de adaptação de cultivares (GALMARINI, 1997).

Em plantas conduzidas sob condições ideais, a bulbificação é acelerada com alta densidade de plantas (RABINOWITCH; BREWSTER, 1990). Estudando os espaçamentos de $10 \times 15 \mathrm{~cm}$ e $20 \times 15 \mathrm{~cm}$, Viegas D'Abreu (1996), verificou que a maior produtividade foi obtida no menor espaçamento $(10 \times 15 \mathrm{~cm})$, e que a maior massa fresca do bulbo (145,7 $\mathrm{g}$ bulbo $\left.^{-1}\right)$ foi verificada no maior espaçamento comparado aos $118 \mathrm{~g} \mathrm{bulbo}^{-1}$ obtido com $10 \times 15 \mathrm{~cm}$.
A produtividade comercial aumentou e a massa fresca do bulbo diminuiu quando o número de linhas por canteiro passou de duas para quatro e o espaçamento entre plantas reduziu de 21,9 para 7,6 $\mathrm{cm}$. A maior porcentagem de bulbos pequenos e médios foi verificada nos menores espaçamentos (STOFFELLA, 1996). Kanton et al. (2002) observaram aumento na produtividade com o incremento da densidade de plantio (37,04 para 156,25 plantas $\left./ \mathrm{m}^{2}\right)$, assim como menor altura de planta e massa fresca do bulbo, relatando que densidades acima de 76,92 plantas por metro quadrado como as que proporcionam maiores produtividades de bulbos comerciais. Lopes et al. (2004), avaliando a influência da densidade de plantas sobre a produtividade 
em três espaçamentos $(0,20 ; 0,30$ e 0,40 $\mathrm{m}$ x $0,08 \mathrm{~m})$, constataram que no menor espaçamento $(0,20 \times 0,08 \mathrm{~m})$ foi obtida a maior produtividade comercial e menor diâmetro do bulbo.

$\mathrm{O}$ incremento na densidade de plantio proporcionando aumento na produtividade total e redução do tamanho do bulbo é também relatado por outros autores (BREWSTER; SALTER, 1980; HARTRIDGE-ESH; BENNET, 1980; MCGEARY, 1985). Por outro lado, Sabota e Downes (1981) não observaram diferenças significativas na produtividade quando compararam duas populações de plantas (192.940 e 257.320 plantas ha $^{-1}$ ), na cultivar Texas Grano. Da mesma forma, avaliando duas densidades de plantio (7,5 cm entre plantas e 12,5 e 15,0 cm entrelinhas), Aujla e Madan (1992) não encontraram diferenças na produtividade e na altura da planta. Entretanto, o maior espaçamento aumentou o número de folhas e o diâmetro transversal do bulbo.

A região Nordeste, representada pelos estados de Pernambuco e Bahia, privilegiada pelas suas condições climáticas, pratica a semeadura de janeiro a dezembro, com maior concentração de plantio nos meses de janeiro a março, possibilitando escalonamento de plantio e produção com oferta em diferentes períodos. No entanto, comparativamente ao primeiro semestre ocorrem menores produtividades no segundo semestre. Todavia, com o lançamento da cultivar Alfa Tropical recomendada para plantio de verão e no segundo semestre na região, sob condições de temperatura elevada e fotoperíodo crescente (COSTA et al., 2002), vislumbra-se uma nova era para a cebolicultura no Nordeste, pela possibilidade de maior produção na entressafra, e possivelmente viabilizando a substituição da importação do produto (MENDONÇA, 2001).

Procurando fornecer maiores informações técnicas sobre o cultivo da cebola Alfa Tropical no segundo semestre do ano, no presente trabalho avaliou-se o efeito de diferentes espaçamentos sobre as características produtivas e de armazenamento dos bulbos, nas condições do Vale do São Francisco.

\section{MATERIAL E MÉTODOS}

O experimento foi conduzido de setembro de 1999 a março de 2000, na Embrapa Semi-Árido em Petrolina, a $9^{\circ}$
24' de latitude Sul e 40 29' de longitude Oeste, e $365,5 \mathrm{~m}$ de altitude. O solo classificado como Latossolo Vermelho Amarelo Distrófico apresentou $\mathrm{pH}$ $\left(\mathrm{H}_{2} \mathrm{O}\right)=6,1 ; \mathrm{Ca}=2,1 \mathrm{cmol} / \mathrm{dm}^{3} ; \mathrm{Mg}=$ $0,7 \mathrm{cmol}_{\mathrm{c}} / \mathrm{dm}^{3} ; \mathrm{Na}=0,01 \mathrm{cmol}_{\mathrm{c}} \mathrm{dm}^{-3} ; \mathrm{K}$ $=0,28 \mathrm{cmol} \mathrm{dm}^{-3} ; \mathrm{Al}=0,05 \mathrm{cmol} \mathrm{dm}^{-3}$, $\mathrm{P}($ Mehlich $)=7,2 \mathrm{mg} \mathrm{dm}^{-3}$ e M.O. $=6,9$ $\mathrm{g} \mathrm{kg}^{-1}$.

$\mathrm{O}$ delineamento experimental utilizado foi de blocos ao acaso, em esquema fatorial $2 \times 3$, compreendendo dois espaçamentos entrelinhas $(0,10$ e 0,15 m) e três espaçamentos entre plantas $(0,10 ; 0,20$ e $0,30 \mathrm{~m})$ com quatro repetições. A unidade experimental constituiu-se de um canteiro de 3,0 $\mathrm{m}$ de comprimento por $1,2 \mathrm{~m}$ de largura, sendo utilizada como área útil 1,8 $\mathrm{m}^{2}(3,0 \mathrm{x}$ $0,6 \mathrm{~m}$ ). A adubação de plantio foi realizada com $600 \mathrm{~kg} \mathrm{ha}^{-1}$ da fórmula NPK 6-24-12, baseada no resultado da análise do solo. Foram aplicados em cobertura $200 \mathrm{~kg} \mathrm{ha}^{-1}$ de uréia e $50 \mathrm{~kg} \mathrm{ha}^{-1} \mathrm{de}$ cloreto de potássio, parcelados aos 15 e 30 dias após o transplante.

Foi utilizada a cultivar Alfa Tropical, desenvolvida pela Embrapa Hortaliças, recomendada para plantio de verão, sendo a semeadura feita em 24/09/ 1999 e o transplante efetuado 30 dias após.

O preparo do solo constou de aração, gradagem e levantamento dos canteiros a $0,20 \mathrm{~m}$ de altura. A cultura foi mantida no limpo através de capinas manuais. A irrigação por microaspersão foi realizada três vezes por semana, com lâminas em torno de $10 \mathrm{~mm}$, baseada na evaporação do tanque classe $\mathrm{A}$, e suspensas 20 dias antes da colheita. Os demais tratos fitossanitários foram realizados de acordo com as recomendações para a cultura da cebola.

A colheita foi realizada em 100 dias após a semeadura quando $70 \%$ das plantas estavam estaladas, com sinais avançados de senescência, como amarelecimento e seca das folhas. A cura foi realizada ao sol por três dias e 12 dias à sombra em galpão ventilado.

Foram avaliadas a produtividade comercial de bulbos (bulbos perfeitos e com diâmetro transversal $>35 \mathrm{~mm}$ ) e refugos (bulbos com diâmetro $<35 \mathrm{~mm}$ ) expressos em $\mathrm{t} \mathrm{ha}^{-1}$, aos 15 dias após a cura. A massa fresca de bulbo ( $\mathrm{g}$ bulbo ${ }^{-1}$ ) foi determinada dividindo-se o peso de bulbos comerciais após a cura pelo número de bulbos. Após o período de cura, os bulbos foram armazenados à temperatura ambiente e realizadas pesagens aos 20; 40 e 60 dias (temperatura e umidade relativa médias no período de $27,2^{\circ} \mathrm{C}$ e $74 \%$, respectivamente), sendo os valores comparados àqueles obtidos ao final da cura (15 dias após colheita). Os valores foram transformados em porcentagem de perda de massa fresca. A classificação de bulbos comerciais segundo o diâmetro transversal ( $\mathrm{mm}$ ) foi feita de acordo com Brasil (1995) em classe 2 (>35 até $50 \mathrm{~mm}$ de diâmetro); classe 3 (>50 até $70 \mathrm{~mm}$ ); classe 4 (>70 ate $90 \mathrm{~mm}$ ) e classe 5 (>90 $\mathrm{mm})$; , sendo os resultados expressos em porcentagem.

Segundo a metodologia descrita por Pimentel Gomes (2000), os dados coletados foram submetidos à análise de variância e as médias comparadas pelo teste de Tukey, ao nível de 5\% de probabilidade, e regressão polinomial. Os dados de porcentagem foram transformados em arco-seno $\sqrt{P / 100}$ para efeitos de análise, sendo apresentados nos resultados as médias originais.

\section{RESULTADOS E DISCUSSÃO}

Efeitos significativos para espaçamentos entrelinhas e entre plantas, assim como para a interação foram obtidos para produtividade comercial. Verificou-se reduções lineares na produtividade dos espaçamentos entre linhas com o aumento do espaçamento entre plantas (Figura 1). Desdobrandose a interação em função do espaçamento entre linhas observou-se maior produtividade média $\left(47,05 \mathrm{t} \mathrm{ha}^{-1}\right)$ do espaçamento $0,10 \mathrm{~m}$ entrelinhas comparativamente ao espaçamento de 0,15 m (41,11 $\left.\mathrm{t} \mathrm{ha}^{-1}\right)$ nos diferentes espaçamentos entre plantas. A população de plantas ideal a ser empregada é aquela suficiente para atingir o índice de área foliar ótimo a fim de interceptar o máximo de radiação solar útil à fotossíntese e ao mesmo tempo maximizar a fração da matéria seca alocada às partes vegetativas e produtivas (HAO; PAPADOPOULOS, 1999), diminuindo as pressões de competição 


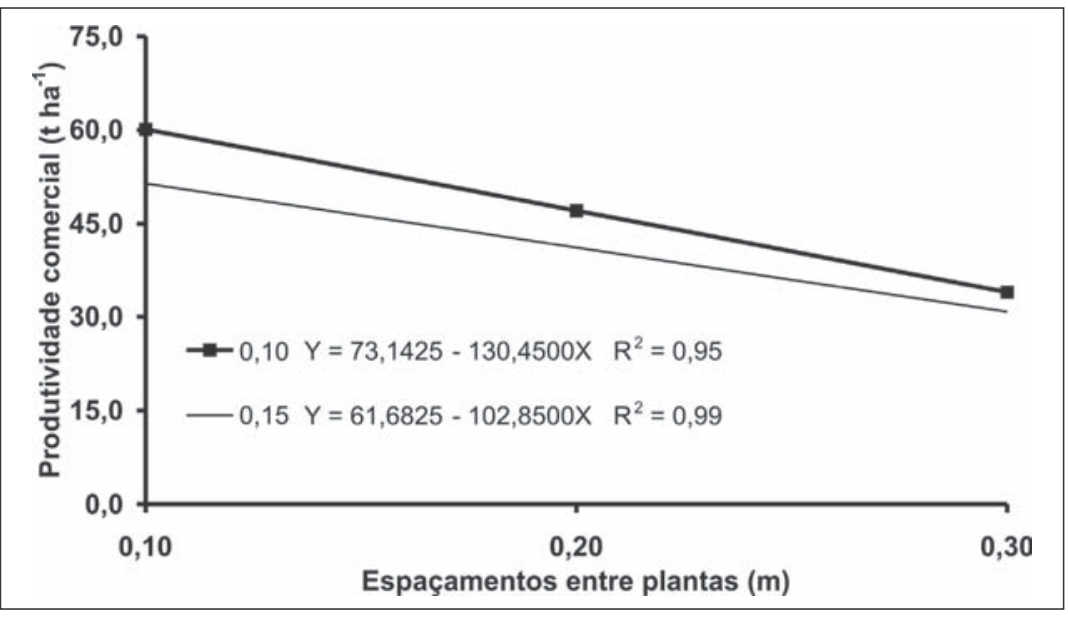

Figura 1. Produtividade comercial de bulbos de cebola, cv. Alfa Tropical, nos espaçamentos 0,10 e 0,15 m entrelinhas em função dos espaçamentos entre plantas. Petrolina, Embrapa Semi-Árido, 1999/2000.

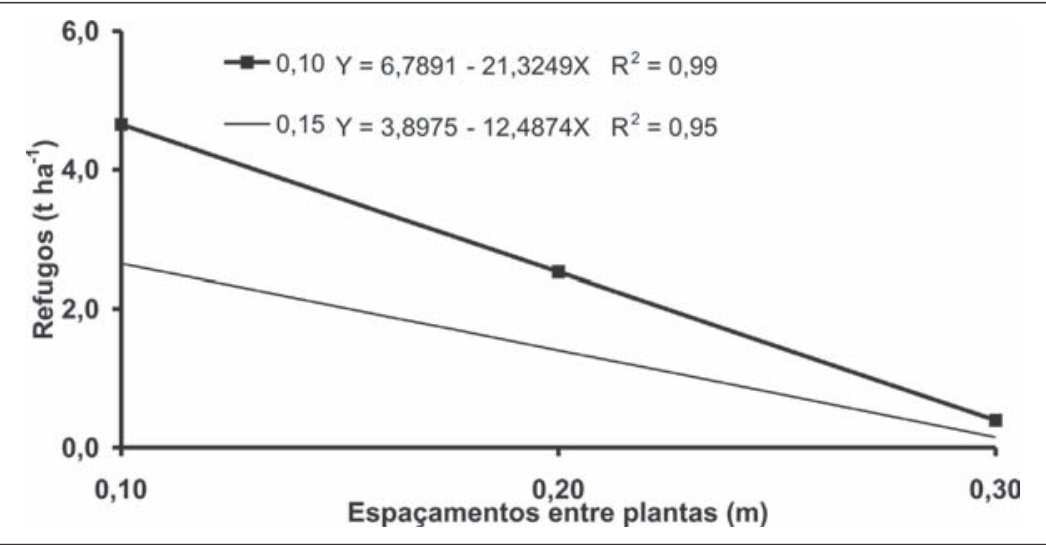

Figura 2. Refugos (produtividade não comercial) de bulbos de cebola, cv. Alfa Tropical, nos espaçamentos 0,10 e $0,15 \mathrm{~m}$ entrelinhas em função dos espaçamentos entre plantas. Petrolina-PE, Embrapa Semi-Árido, 1999/2000.

interplantas. Os resultados obtidos confirmam as observações de que as cebolas respondem especialmente à variação no espaçamento entre plantas (FILGUEIRA, 1982; MELO et al., 1988). A produtividade da cebola também aumentou à medida que se diminuiu à distância entrelinhas, fato também relatado por diversos autores em diferentes épocas (MANGUAL-CRESPO et al., 1979; LOPES, 1987; STOFFELLA, 1996; BOFF et al., 1998).

Resultados similares foram obtidos no que se refere à produção de refugo (bulbos não comerciais), ou seja, verificou-se uma redução linear gradativa na produção de bulbos não comerciais à medida que se aumentou o comparativamente ao cultivo em densidade adequada.

Notou-se para massa fresca do bulbo efeito significativo da interação dos fatores. Foi verificado que a massa fresca do bulbo aumentou linearmente à medida que se aumentaram os espaçamentos entre plantas. Este incremento foi da ordem de $34,9 \%$ e $55,91 \%$, respectivamente, para os espaçamentos de 0,10 e 0,15 m entrelinhas (Figura 3). No que se refere aos espaçamentos entrelinhas constatou-se bulbos com maiores médias de massa fresca no espaçamento de $0,15 \mathrm{~m}(102,57 \mathrm{~g}$ bulbo $\left.^{-1}\right)$ comparativamente ao espaçamento de $0,10 \mathrm{~m}\left(85,88 \mathrm{~g}^{\text {bulbo-1 }}{ }^{-1}\right)$, dentro dos espaçamentos entre plantas. Salienta-se que a maior massa fresca do bulbo foi obtida tanto com o aumento do espaçamento entre plantas quanto com o espaçamento entrelinhas, o que, está relacionado à maior área de exploração das raízes e menor competição interplantas por água, luz e nutrientes. $\mathrm{O}$ incremento da densidade, reduzindo o diâmetro e a massa fresca do bulbo é relatada por Lopes (1987) e Lopes et al. (2004). A diminuição da massa fresca do bulbo com o incremento da densidade de plantio foi também observada por outros autores (GALMARINI; GASPERA, 1995; STOFFELLA, 1996; LIPINSKI et al., 2002; KANTON et al., 2002). Segundo Souza e Resende (2002), o mercado consumidor nacional prefere bulbos de tamanho médio com pesos de 80 a 100 gramas e diâmetro transversal de 40 a $80 \mathrm{~mm}$, que se enquadram perfeitamente dentro das faixas de tamanho de bulbo obtidas no presente estudo.

A classificação de bulbos de cebola foi influenciada pela interação entre os fatores (Tabela 1). No que se refere à classificação de bulbos classe 2, constatou-se para os espaçamentos de 0,10 e $0,15 \mathrm{~m}$ entrelinhas reduções lineares deste tipo de bulbo com o aumento do espaçamento entre plantas, que são menores comparativamente às demais classes. Com relação ao desdobramento da interação no sentido do espaçamento entrelinhas verificou-se que o espaçamento de 0,10 apresentou maior número de bulbos médios nesta classe dentro dos diferentes espaçamentos 
$(33,45 \%)$ quando comparado ao espaçamento de $0,15 \mathrm{~m}(23,12 \%)$.

Resultados inversos foram verificados para a classe 3 (bulbos de tamanho intermediário), pois, ocorreram aumentos lineares com o incremento do espaçamentos entre plantas. Desdobrando-se a interação, não se observou para os maiores espaçamentos entre plantas $(0,20$ e $0,30 \mathrm{~m})$ diferenças significativas dentro dos espaçamentos de 0,10 e 0,15 m entrelinhas; fato este que só foi verificado quando da utilização do menor espaçamento entre plantas $(0,10 \mathrm{~m})$, que proporcionou maior percentagem de bulbos desta classe no espaçamento de $0,10 \mathrm{~m}$ entre linhas $(54,43 \%)$ comparativamente a $0,15 \mathrm{~m}$ entrelinhas $(49,20 \%)$.

Para a classe 4 , composta de bulbos de maior tamanho, registrou-se efeitos semelhantes (Tabela 1) aos anteriores ocorrendo aumentos lineares com o incremento do espaçamentos entre plantas. No entanto, desdobrando-se em função dos espaçamentos entrelinhas as maiores percentagens médias de bulbos desta classe foram obtidas no espaçamento de $0,15 \mathrm{~m}$ entrelinhas $(24,14 \%)$ comparado ao espaçamento de $0,10 \mathrm{~m}(10,82 \%)$. Estes resultados demonstram o efeito da densidade de plantio como fator determinante na produção de cebola ao mesmo tempo que corroboram com os obtidos por Viegas D'Abreu (1996), que obteve maior tamanho de bulbo no maior espaçamento, assim como os obtidos por Stoffella (1996) que verificou maior porcentagem de bulbos pequenos e médios nos menores espaçamentos, e Rumpel e Felczynski (2000) que encontraram redução na produção de bulbos maiores com o incremento da densidade de plantio.

As pressões exercidas pela população de plantas afetam de modo marcante o desenvolvimento da cebola. Os resultados obtidos no presente trabalho evidenciaram uma relação inversa entre a densidade de plantio e o tamanho do bulbo. De forma geral, altas densidades produzem maior número bulbos por área, mas com menor massa fresca e consequentemente menor produtividade comercial; fato este atribuído principalmente às pressões de competição interplantas. Quando a densidade de

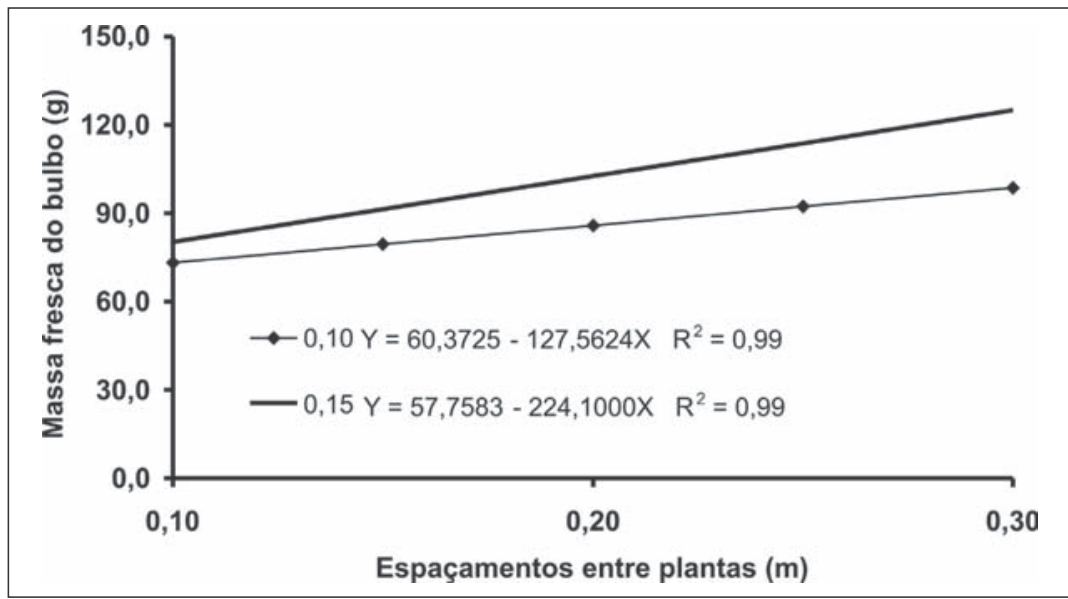

Figura 3. Massa fresca do bulbo de cebola, cv. Alfa Tropical, nos espaçamentos 0,10 e 0,15 $\mathrm{m}$ entrelinhas em função dos espaçamentos entre plantas. Petrolina-PE, Embrapa SemiÁrido, 1999/2000.

Tabela 1. Equações de regressão para classificação de bulbos de cebola, cv. Alfa Tropical, em classes (\%), segundo o diâmetro transversal nos espaçamentos 0,10 e 0,15 m entrelinhas em função dos espaçamentos 0,10; 020 e 0,30 m entre plantas. Petrolina-PE, Embrapa SemiÁrido, 1999/2000.

\begin{tabular}{lll}
\hline Classes $^{1}$ & \multicolumn{1}{c}{ Equações de regressão } \\
\hline \multirow{2}{*}{ classe 2 } & $\mathrm{Y}(0,10 \mathrm{~m})=49,9067-72,8530^{* *} \mathrm{X}$ & $\mathrm{R}^{2}=0,95$ \\
& $\mathrm{Y}(0,15 \mathrm{~m})=59,9518-156,0420^{* *} \mathrm{X}$ & $\mathrm{R}^{2}=0,95$ \\
\hline \multirow{2}{*}{ classe 3 } & $\mathrm{Y}(0,10 \mathrm{~m})=41,2463+31,4903^{* *} \mathrm{X}$ & $\mathrm{R}^{2}=0,90$ \\
& $\mathrm{Y}(0,15 \mathrm{~m})=32,0498+62,4829^{* *} \mathrm{X}$ & $\mathrm{R}^{2}=0,85$ \\
\hline \multirow{2}{*}{ classe 4 } & $\mathrm{Y}(0,10 \mathrm{~m})=5,8051+67,0393^{* *} \mathrm{X}$ & $\mathrm{R}^{2}=0,94$ \\
& $\mathrm{Y}(0,15 \mathrm{~m})=11,4103+90,1114^{* *} \mathrm{X}$ & $\mathrm{R}^{2}=0,93$ \\
\hline
\end{tabular}

** Significativo ao nível de $1 \%$ de probabilidade pelo teste de $\mathrm{F}$; ${ }^{1}$ classe $2:>35$ até $50 \mathrm{~mm}$ de diâmetro; classe 3: >50 até $70 \mathrm{~mm}$ e classe 4: >70 ate $90 \mathrm{~mm}$.

plantas aumenta por unidade de área, atinge-se um ponto no qual as plantas competem por fatores essenciais de crescimento, como nutrientes, luz e água.

A perda de peso aos 20; 40 e 60 dias após cura (DAC) apresentou efeitos significativos apenas para o espaçamento entre plantas, não se constatando efeito significativo para o espaçamento entrelinhas e nem para a interação dos fatores. Para perda de peso aos $20(\mathrm{Y}=$ $\left.7,5386+12,8168 * * \mathrm{X} ; \mathrm{R}^{2}=0,99\right), 40$ $\left(\mathrm{Y}=17,7093+10,8710 * * \mathrm{X} ; \mathrm{R}^{2}=0,92\right)$ e $60\left(\mathrm{Y}=16,0176+32,4786 * * \mathrm{X} ; \mathrm{R}^{2}=\right.$ $0,99)$ dias após cura, verificou-se aumentos lineares evidenciando maiores perdas de peso com o incremento dos espaçamentos. Pelos coeficientes angulares das equações de regressão, estimase esta perda de massa em 1,28, 1,09 e $3,25 \%$ para cada aumento do espaçamento entre plantas $(0,10 \mathrm{~m})$ para
20, 40 e 60 DAC. Inferiu-se por estes resultados, que a maior perda ocorreu em espaçamentos maiores, que determinaram maior tamanho de bulbos, com consequiente maior teor de água nos bulbos. Calbo et al. (1980) observaram perdas de $30 \%$ a $100 \%$ em bulbos de cebola Baia Periforme armazenadas até 70 dias, a granel e réstias em diferentes recipientes, assim como Resende et al. (2004) informam para a cultivar Texas Grano 502 PPR que o espaçamento de $0,30 \times 0,15 \mathrm{~m}$ foi o que promoveu maior percentagem de perda de peso de bulbos $(43,16 \%)$, sob as condições do Vale do São Francisco, armazenadas até 60 dias após a cura.

Pelos resultados obtidos em função das diferentes características avaliadas, recomenda-se os espaçamentos de 0,10 x 0,15 m entrelinhas e 0,10 m entre plantas como os mais adequados para o cul- 
tivo da cebola cultivar Alfa Tropical, nas condições do Vale do São Francisco, para plantio no segundo semestre do ano.

\section{LITERATURA CITADA}

AUJLA, T.S.; MADAN, P.S. Response of onion (Allium cepa) to irrigation, nitrogen fertilizer and row spacing on deep sandy-loam in subtropical monsoon region. Indian Journal of Agricultural Science, v.62, n.2, p.129-134, 1992.

BOFF, P., HENRI, S.; GONÇALVES, P.A.S. Influência da densidade de plantas na ocorrência de doenças foliares e produção de bulbos de cebola. Fitopatologia Brasileira, Brasília, v.23, n.4, p.448 452, 1998.

BRASIL. Ministério da Agricultura, Abastecimento e Reforma Agrária. Portaria n.529 de 18 ago. 1995. Diário Oficial da República Federativa do Brasil, Brasília, 1 set.1995, Seção1, p.13513.

BREWSTER, J.L., SALTER, P.J. The effect of plant spacing on the yield and bolting of two cultivars of overwintered bulb onions. Journal of Horticultural Science, v.55, n.2, p.97-102, 1980. CALBO, A.G.; CORDEIRO, C.M.T.; GUALBERTO, A.G. Efeito do acondicionamento sobre o comportamento fisiológico e conservação de duas cultivares de cebola (Allium cepa) no Vale do São Francisco. Revista de Olericultura, Brasília, v.18, p.86-99, 1980.

COSTA, N.D.; LEITE, D.L.; SANTOS, C.A.F.; CANDEIA, J.A.; VIDIGAL, S.M. Cultivares de cebola. Informe Agropecuário, Belo Horizonte, v.23, n.218, p.20-27, 2002.

FAO. Agricultural production, primary crops. Disponível em <http://www.fao.org >. Acesso em: 26 jan. 2005.

FILGUEIRA, F.A.R. Manual de Olericultura: cultura e comercialização de hortaliças. 2. ed., São Paulo: Agronômica Ceres, 1982. v.2, 357 p. HAO, X.; PAPADOPOULOS, A.P. Effects of supplemental lighting and cover materials on growth, photosynthesis, biomass partitioning, early yield and quality of greenhouse cucumber. Scientia Horticulturae, v.80, n.1-2, p.1-18, 1999.
GALMARINI, C.R. Caracteristicas botanicas y fisiologicas. In: Manual del cultivo de la cebolla. GALMARINI, C.R. (ed.). San Juan: INTA, 1997. $128 \mathrm{p}$.

GALMARINI, C.R.; GASPERA, P.G. Efecto de la epoca de transplante y la densidad de plantacion en el cultivo de cebolla tipo Valenciana. Horticultura Argentina, v.14, n.37. p.23-29, 1995. HARTRIDGE-ESH, K.A.; BENNET, J.P. Effects of seed weight plant density and spacing on yield response of onion. Journal of Horticultural Science, v.55, n.3, p.247-252, 1980.

INSTITUTO BRASILEIRO DE GEOGRAFIA E ESTATÍSTICA - IBGE. Produção Agrícola $M u$ nicipal. Disponível em <http:// www.sidra.ibge.gov.br>. Acesso em: 26 jan. 2005. KANTON, R.A.L; ABBEY, L.; HILLA, R.G.; TABIL, M.A.; JAN, N.D. Density affects plant development and yield of bulb onion (Allium cepa L.) in Northern Ghana. Journal Vegetable Crop Production, v.8, n.2, p.5-25, 2002.

LIPINSKI, V.M., GAVIOLA, S.; GAVIOLA, J.C. Efecto de la densidad de plantación sobre el rendimiento de cebolla cv. Cobriza Inta con riego por goteo. Agricultura Técnica, v.62,n.4, p.574582, 2002.

LOPES, J.F. Effects of planting dates and spacing on several characteristics of short day onion varieties grown in South Texas. 1987. 111 f. (Tese doutorado) - Texas University, Texas.

LOPES, M.C.; CZEPAK, M.P.; SIRTOLI, L.F. Avaliação de diferentes espaçamentos na produtividade de três cultivares de cebola. Horticultura Brasileira, Brasília, v.22, n.2, julho 2004, Suplemento 2. CD-ROM. Trabalho apresentado no $44^{\circ}$ Congresso Brasileiro de Olericultura, 2004.

MANGUAL-CRESPO, G.; RAMIREZ, C.T.; ORENGO, E. Effect of plant spacing and fertilizer levels on yield and dry bulb weight of onion cv. Texas Grano 502. Journal of Agriculture of the University of Puerto Rico, v.63, n.4, p.417-422, 1979.

MCGEARY, D.F. The effect of plant density on shape, size, uniformity, soluble solids content and yield of onions suitable for pickling. Journal Horticultural Science, v.60, n.1, p.83-87, 1985.
MELO, P.C.T.; RIBEIRO, A. Produção de sementes de cebola: cultivares de polinização aberta e híbridos. ln: CASTELLANE; P.D.; NICOLOSI, W.M.; HASEGAWA, M. Produção de sementes de hortaliças. Jaboticabal: FCAV/FUNEP, 1990. p.15-59.

MELO, P.C.T.; RIBEIRO, A.; CHURATA-MASCA, M.G.C. Sistemas de produção, cultivares de cebola e seu desenvolvimento para as condições brasileiras. In: SEMINÁRIO NACIONAL DE CEBOLA, 3, 1988, Piedade-SP. Anais... Jaboticabal: FUNEP, 1988. p.27-61.

MENDONÇA, J.L. Cultivar de cebola de verão. Disponível em: 〈http//www.snagricultura.org.br>. Acesso em: 29 ago. 2001.

PIMENTEL GOMES, F. Curso de estatística experimental. 14.ed. São Paulo: Nobel, 2000. 477 p.

RABINOWITCH, H.D.; BREWSTER, J.L. Onions and Allied Crops. Boca Raton: CRC Press, 1990. 273 p.

RESENDE, G.M.; COSTA, N.D.; ALVARENGA, M.A.R. Características produtivas e conservação pós-colheita da cebola (Allium Cepa L.) cv. Texas Grano PRR em diferentes espaçamentos de plantio. Horticultura Brasileira, Brasília, v.22, n.2 julho 2004, Suplemento 2. CD-ROM. Trabalho apresentado no $44^{\circ}$ Congresso Brasileiro de Olericultura, 2004.

RUMPEL, J.; FELCZYNSKI, K. Effect of plant density on yield and bulb size of direct sown onions. Acta Horticulturae, n.533, p.179-186, 2000.

SABOTA, C.M.; DOWNES, J.D. Onion growth and yield in relation to transplant, pruning, size, spacing and depth of planting. Hortscience, v.16, n.4, p.533-535, 1981.

SOUZA, R.J., RESENDE, G.M. Cultura da cebola. Lavras: UFLA, 2002. 115 p. (Textos Acadêmicos - Olericultura, 21).

STOFFELLA, P.J. Planting arrangement and density of transplants influence sweet Spanish onion yields and bulb size. Hortscience, v.31, n.7, p.1129-1130, 1996

VIEGAS D'ABREU, D.A. Effect of nitrogen and spacing on bulb splitting in onion cv. Bombay Red. TVIS Newsletter, v.1, n.2, p.26, 1996. 